\title{
Diagnosing COVID-19 pneumonia in a pandemic setting: Lung Ultrasound versus CT (LUVCT) - a multicentre, prospective, observational study
}

\author{
Arthur W.E. Lieveld (1) ${ }^{1,6}$, Bram Kok ${ }^{2,6}$, Frederik H. Schuit ${ }^{1,6}$, Kaoutar Azijli ${ }^{3}$, \\ Jarom Heijmans ${ }^{4}$, Arjan van Laarhoven ${ }^{2}$, Natascha L. Assman², Ruud S. Kootte ${ }^{4}$, \\ Tycho J. Olgers ${ }^{5}$, Prabath W.B. Nanayakkara (1), ${ }^{1}$ and Frank H. Bosch ${ }^{2}$
}

\section{ABSTRACT}

Background: In this coronavirus disease 2019 (COVID-19) pandemic, fast and accurate testing is needed to profile patients at the emergency department (ED) and efficiently allocate resources. Chest imaging has been considered in COVID-19 workup, but evidence on lung ultrasound (LUS) is sparse. We therefore aimed to assess and compare the diagnostic accuracy of LUS and computed tomography (CT) in suspected COVID-19 patients.

Methods: This multicentre, prospective, observational study included adult patients with suspected COVID-19 referred to internal medicine at the ED. We calculated diagnostic accuracy measures for LUS and CT using both PCR and multidisciplinary team (MDT) diagnosis as reference. We also assessed agreement between LUS and CT, and between sonographers.

Results: One hundred and eighty-seven patients were recruited between March 19 and May 4, 2020. Area under the receiver operating characteristic (AUROC) was 0.81 (95\% CI $0.75-0.88$ ) for LUS and 0.89 ( $95 \%$ CI 0.84-0.94) for CT. Sensitivity and specificity for LUS were 91.9\% (95\% CI 84.0-96.7) and 71.0\% (95\% CI 61.1-79.6), respectively, versus $88.4 \%$ (95\% CI 79.7-94.3) and $82.0 \%$ (95\% CI 73.1-89.0) for CT. Negative likelihood ratio was 0.1 (95\% CI $0.06-0.24)$ for LUS and 0.14 (95\% CI $0.08-0.3$ ) for CT. No patient with a false negative LUS required supplemental oxygen or admission. LUS specificity increased to $80 \%$ (95\% CI 69.9-87.9) compared to MDT diagnosis, with an AUROC of 0.85 (95\% CI 0.79-0.91). Agreement between LUS and CT was 0.65. Interobserver agreement for LUS was good: 0.89 (95\% CI 0.83-0.93).

Conclusion: LUS and CT have comparable diagnostic accuracy for COVID-19 pneumonia. LUS can safely exclude clinically relevant COVID-19 pneumonia and may aid COVID-19 diagnosis in high prevalence situations.

@ERSpublications

The \#luvct study found that LUS and CT have comparable diagnostic accuracy for \#COVID19 pneumonia. LUS safely excludes COVID-19 pneumonia and may aid diagnosis of COVID-19. This simple tool may prove especially useful in resource constrained settings. https://bit.ly/33P71fq

Cite this article as: Lieveld AWE, Kok B, Schuit FH, et al. Diagnosing COVID-19 pneumonia in a pandemic setting: Lung Ultrasound versus CT (LUVCT) - a multicentre, prospective, observational study. ERJ Open Res 2020; 6: 00539-2020 [https://doi.org/10.1183/23120541.00539-2020].

This article has supplementary material available from openres.ersjournals.com

This study is registered at trialregister.nl with identifier number NL8497. Individual participant data that underlie the results reported in this article will be available after deidentification, as well as the study protocol, immediately following publication, to investigators whose proposed use of the data has been approved by an independent review committee, for individual participant data meta-analysis. Proposals should be directed to p.nanayakkara@amsterdamumc.nl; to gain access, data requestors will need to sign a data access agreement.

Received: 30 July 2020 | Accepted after revision: 30 Sep 2020

Copyright $\odot$ ERS 2020. This article is open access and distributed under the terms of the Creative Commons Attribution Non-Commercial Licence 4.0. 


\section{Introduction}

Coronavirus disease 2019 (COVID-19) is caused by the novel and rapidly spreading severe acute respiratory syndrome coronavirus 2 (SARS-CoV-2). Cases continue to rise worldwide [1, 2]. With the pandemic hotspot moving to middle- and low-income regions such as Russia, India, Pakistan, Latin America and Africa, the consequences are potentially catastrophic [3]. Since there is no effective treatment yet, early detection is crucial in halting COVID-19.

The SARS-CoV-2 reverse transcriptase polymerase chain reaction (PCR) assay is the gold standard for diagnosing COVID-19. Although it is highly specific, it has limited sensitivity, long turnaround times and there is a worldwide shortage of test capacity. Serological tests are not useful in acute cases, and reliable rapid antigen tests are unfortunately not available $[4,5]$. This hampers immediate triage and decision-making. Moreover, microbiological tests do not give insight into lung involvement, which is the main cause of morbidity and mortality $[6,7]$. Correct assessment of lung involvement is thus crucial for appropriate triage, clinical management and efficient allocation of scarce medical resources.

The World Health Organization (WHO) recently advocated chest imaging, especially when PCR results are not readily available or the initial PCR is negative but clinical suspicion of COVID-19 remains high [8]. However, chest radiography (CXR) sensitivity is low $[9,10]$, and there are differences of opinion on the role of computed tomography (CT) in COVID-19 [9-12].

In view of the unmet clinical need for a fast and reliable test to diagnose or rule out COVID-19 pneumonia, bedside lung ultrasound (LUS) has attracted attention [13-23]. Although many clinicians might be unfamiliar with LUS, its diagnostic properties are better than CXR and physical examination combined, and equivalent to chest $\mathrm{CT}$ in diagnosing acute respiratory pathologies including pneumonia [24-27]. In addition, it has the advantage over CT of being portable, quick, radiation free, easy to disinfect and low cost. Moreover, it integrates real-time imaging into clinical decision-making at the bedside, reducing time to diagnosis and treatment [28-30].

Unfortunately, the literature on the use of LUS in COVID-19 is limited [8]. Therefore, our objective was to investigate the role of LUS in suspected COVID-19 patients at the emergency department (ED). To our knowledge, this is the first prospective study analysing and comparing diagnostic accuracy of LUS and CT in diagnosing pneumonia in patients with suspected COVID-19.

\section{Methods}

\section{Study design and participants}

This is a pragmatic, multicentre, prospective, observational study. Patients were recruited from three academic hospitals in the Netherlands (Radboud University Medical Centre, Nijmegen and both locations of the Amsterdam University Medical Centers) between March 19 and May 4, 2020. On May 4 the percentage of positive PCRs in patients with suspected COVID-19 fell below 10\%. This is used as the epidemic threshold by the Dutch Institute for Research and Healthcare (NIVEL) and National Institute for Public Health and the Environment (RIVM) [31]. The study was registered with the Netherlands Trial Register and approved by the Medical Ethical Committee (CMO region Arnhem-Nijmegen).

All patients 18 years and older who were referred to the ED for internal medicine with suspected COVID-19 were eligible. In our hospitals we adhered to the case definition of the RIVM and WHO. A suspected case of COVID-19 was initially defined as having either fever, malaise, myalgia and respiratory symptoms, but later also included gastrointestinal symptoms, loss of smell or taste and unexplained delirium in the elderly.

Patients were included when CT, LUS and PCR were all performed. Exclusion criteria were age under 18, no verbal consent and uninterpretable CT or LUS. The study protocol has been published and is available online.

Affiliations: 'Section General and Acute Internal Medicine, Dept of Internal Medicine, Amsterdam Public Health Research Institute, Amsterdam UMC, location VUmc, Amsterdam, The Netherlands. ${ }^{2}$ Section Acute Medicine, Dept of Internal Medicine, Radboudumc, Nijmegen, The Netherlands. ${ }^{3}$ Section Emergency Medicine, Emergency Dept, Amsterdam Public Health Research Institute, Amsterdam UMC, location VUmc, Amsterdam, The Netherlands. "Section Acute Medicine, Dept of Internal Medicine, Amsterdam UMC, location AMC Amsterdam, The Netherlands. ${ }^{5}$ Section Acute Medicine, Dept of Internal Medicine, University of Groningen, University Medical Center Groningen, Groningen, The Netherlands. ${ }^{6}$ These authors contributed equally.

Correspondence: Prabath W.B. Nanayakkara, Section General and Acute Internal Medicine, Dept of Internal Medicine, Amsterdam University Medical Center, Location VU University Medical Center, De Boelelaan 1118, 1081 HZ Amsterdam, The Netherlands. E-mail: p.nanayakkaradamsterdamumc.nl 
Medical workup, admission and decision process

All patients received regular medical workup (history, physical examination and routine laboratory tests). In addition, they received a chest CT and/or PCR as indicated by local protocol. CXR was not routinely performed in the workup of suspected COVID-19 in our hospitals. Clinical criteria for admission were: saturation $<94 \%$ and/or respiratory rate $>20 / \mathrm{min}$. It was standard practice in participating hospitals that all admitted patients with suspected COVID-19 were discussed daily by a multidisciplinary team (MDT), consisting at least of consultants in infectious disease, respiratory disease and microbiology. In patients with a high clinical suspicion but negative PCR a diagnosis of COVID-19 could be made by this expert panel on the basis of clinical, laboratory, microbiological and CT data, after excluding alternative diagnoses. No clinical decisions were made based on LUS findings; the MDT was blinded for LUS results.

\section{LUS}

LUS was performed or supervised by internists (mostly registrars) who were certified in point-of-care ultrasound and had performed at least 20 supervised LUS. Both LUS and CT were done at presentation to the ED or within $24 \mathrm{~h}$ of admission. The majority of LUS were performed by the treating physician as an extension of the physical examination. As such, they were not blinded for the patient history or clinical picture. They were blinded for the PCR and CT result.

Handheld ultrasound systems were used with settings amenable to the detection of B-line artefacts (e.g. lung preset, or if this preset was not available, abdominal preset with tissue harmonic imaging switched off and dynamic range put at the lowest level) [16]. See supplementary figure S1 for the scan protocol.

In keeping with pre-specified criteria in recent Chinese, Italian and British literature, LUS was deemed positive if there were three or more B-lines and/or consolidation in two or more zones unilaterally or in one or more zones bilaterally. When COVID-19 features were not found or just in one zone unilaterally, the scan was deemed negative (see supplementary table S1) [15-17, 21, 22]. See supplementary figure S2 and videos for examples of COVID-19 sonographic features.

$C T$

The chest CTs were assessed by the local radiologists. The radiologists were blinded for LUS and PCR results, but not for clinical information. The likelihood of COVID-19 pneumonia was reported via the COVID-19 Reporting and Data System (CO-RADS), which uses a scale from 1 (very low) to 5 (very high), using pre-specified criteria (see supplementary table S2) [32]. A CO-RADS score of 1 or 2 is regarded as negative, a score of 3 is equivocal, and a score of 4 or 5 is deemed positive. For the purpose of this study equivocal CTs were considered negative.

PCR

A PCR test on swab samples obtained from the oropharynx or nasopharynx (or if available, from sputum, faeces, tracheal aspirate or bronchoalveolar lavage) was performed in all patients according to WHO standards. The same PCR assay was used in all participating hospitals. In case of a negative or indeterminate test result but a high clinical suspicion, PCR was repeated. If a patient had an indeterminate test and no PCR was repeated, the PCR was considered negative.

\section{Outcomes}

Following WHO recommendations we used both (serial) PCR (gold standard) and clinical follow-up (MDT diagnosis) to assess the sensitivity, specificity, diagnostic accuracy, area under the receiver operating characteristic (AUROC) curve, predictive values, positive likelihood ratio (PLR) and negative likelihood ratio (NLR) of LUS and CT. Agreement was calculated between LUS and CT. Interobserver agreement was calculated between sonographers.

\section{Statistical analysis}

No sample size calculations were performed for this study. Normally distributed continuous variables are summarised by the mean and standard deviation (SD). Not normally distributed continuous variables are summarised by the median and interquartile range (IQR). Differences between groups were tested using the independent t-test for normally distributed outcomes. Difference in means and $95 \%$ confidence interval were calculated. The widths of the intervals have not been adjusted for multiplicity; inferences drawn may therefore not be reproducible. A non-parametric Mann-Whitney U test was used to compare continuous outcomes that were not normally distributed between groups. Sensitivity and specificity of LUS and CT were compared using the McNemar test.

Discriminatory power of LUS and CT were determined by a receiver operating characteristic (ROC) curve and the corresponding AUROC with 95\% confidence intervals for each cut-off. 
Agreement between LUS and chest CT was quantified with the Cohen's kappa statistic. Interobserver agreement between sonographers was measured with a weighted kappa via the intraclass correlation coefficient with a two-way mixed effects model. Three sonographers independently assessed ultrasound recordings of 60 patients. This number was chosen as it allowed rejection of the null hypothesis that agreement was moderate $(\kappa 0.5)$ in case true agreement was good $(\kappa 0.8)$ with $80 \%$ power. The power calculation was performed assuming proportions of negative and positive ratings of $40 \%$ and $60 \%$. A two-sided significance level of $5 \%$ was used for all analyses. Data were analysed by AL, BK, FS and KA using SPSS version 26 (IBM, Chicago, IL, USA).

\section{Results}

From March 19 until May 4, 2020, 187 patients were recruited for the study (figure 1). There were no missing data or adverse events. PCR positive patients on average had longer duration of symptoms and more often required oxygen therapy and intensive care unit admission than PCR negative patients. See table 1 for patient demographics and clinical characteristics.

\section{Study population}

Seventy-five patients had an initial positive PCR result, while 11 patients turned out positive after repeated testing. Fifteen patients with negative PCR results were eventually diagnosed with COVID-19 (table 2 and supplementary figures S3 and S4). The most common alternative diagnoses in the PCR negative group were bacterial/aspiration pneumonia (14\%), upper respiratory tract infection (11\%), progression of malignant disease (9\%), exacerbation asthma/COPD (7\%) and decompensated heart failure $(7 \%)$ (supplementary table S3).

\section{Diagnostic performance}

For LUS we found a sensitivity of $91.9 \%$ (95\% CI 84.0-96.7) and a specificity of $71.0 \%$ (95\% CI 61.1-79.6) compared to PCR as a reference standard. AUROC was 0.81 (95\% CI 0.75-0.88). The PLR and NLR were 3.2 (95\% CI 2.3-4.3) and 0.1 (95\% CI 0.06-0.24), respectively.

Comparing CT to PCR as a reference standard, we found a sensitivity of $88.4 \%$ (95\% CI 79.7-94.3) and specificity of $82.0 \%$ (95\% CI 73.1-89.0). AUROC was 0.89 (95\% CI 0.84-0.94). The PLR and NLR were 4.9 (95\% CI 3.2-7.5) and 0.14 (95\% CI 0.08-0.26), respectively. See tables 2 and 3 for full results, supplementary figures S5-S7 for ROC curves and supplementary table S4 for details on false negative LUS and CT.

When we compared LUS to the MDT diagnosis, the AUROC increased to 0.85 (95\% CI 0.79-0.91). Specificity increased to $80.0 \%$ (95\% CI 69.9-87.9) and PLR to 4.5 (95\% CI 2.9-6.9), while the NLR remained similar at $0.12(95 \% \mathrm{CI} 0.07-0.2)$.

Using both PCR and the MDT diagnosis as a reference, the McNemar test found no significant difference in sensitivity or specificity between LUS and CT (supplementary table S5). The agreement between LUS and chest CT was substantial: 0.65 . The interobserver agreement between scanning physicians was good: 0.89 (95\% CI 0.83-0.93).

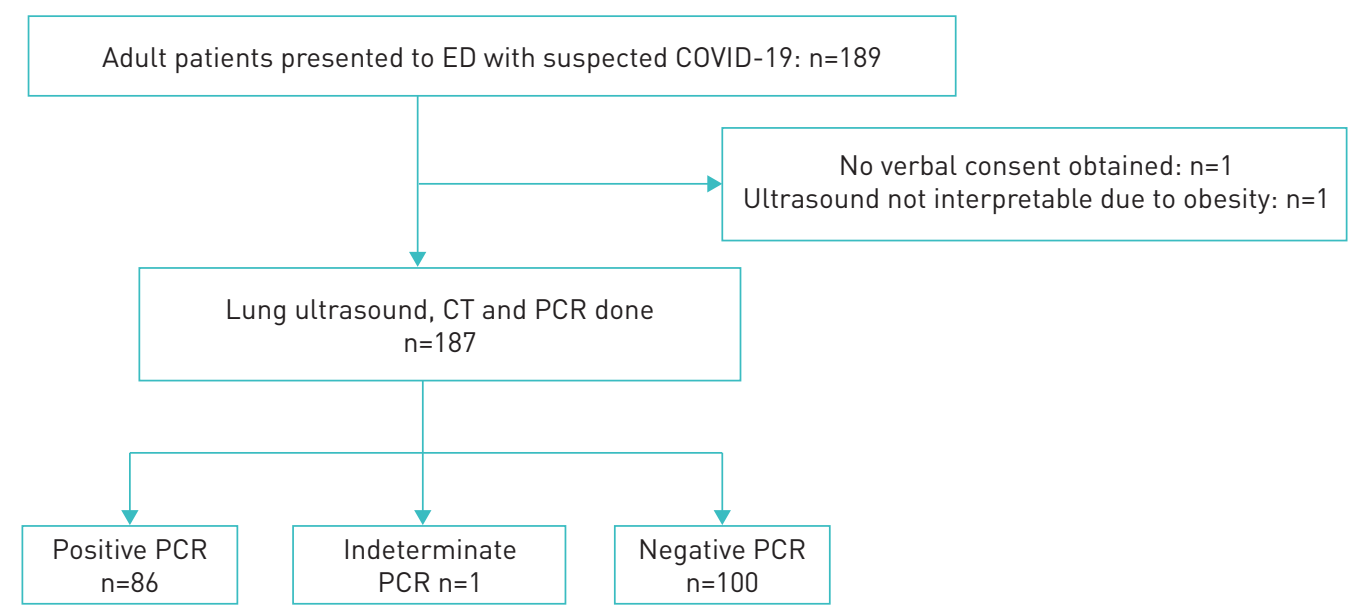

FIGURE 1 Study population flowchart. ED: emergency department; CT: computed tomography. 


\begin{tabular}{|c|c|c|c|c|}
\hline & All patients & PCR positive & PCR negative & p-value \\
\hline Subjects $\mathrm{n}$ & $187^{\#}$ & 86 & 100 & \\
\hline Age years & $63.7 \pm 15.7$ & $63.4 \pm 14.8$ & $64.1 \pm 16.5$ & 0.668 \\
\hline Male & $108(57.8)$ & $50(58.1)$ & 58 (58.0) & 0.97 \\
\hline Admission & $140(74.9)$ & $65(75.6)$ & $75(75.0)$ & 0.855 \\
\hline Admission ED to IC & $9(4.8)$ & $8(9.3)$ & $1(1.0)$ & $<0.001$ \\
\hline In hospital mortality & $9(4.8)$ & $5(5.8)$ & $4(4.0)$ & 0.001 \\
\hline Obesity (based on clinical assessment) & $52(27.8)$ & $27(31.4)$ & $25(25)$ & 0.676 \\
\hline Duration of symptoms days & $6.5 \pm 5.1$ & $7.3 \pm 4.1$ & $5.8 \pm 5.8$ & 0.007 \\
\hline \multicolumn{5}{|l|}{ Comorbidities } \\
\hline Asthma & 10 (5.3) & 3 (3.5) & 7 (7.0) & 0.033 \\
\hline Chronic cardiovascular disease & $44(23.5)$ & $18(20.9)$ & $26(26.0)$ & 0.104 \\
\hline COPD (GOLD stage >2) & $30(5.3)$ & $12(14.0)$ & $18(18.0)$ & 0.134 \\
\hline Current malignancy & 35 (18.7) & $9(10.5)$ & $26(26.0)$ & $<0.001$ \\
\hline Diabetes mellitus & 36 (19.3) & $21(24.4)$ & $15(15.0)$ & 0.001 \\
\hline \multicolumn{5}{|l|}{ Laboratory analysis on admission } \\
\hline CRP $\mathrm{mg} \cdot \mathrm{L}^{-1}$ median (interquartile range) & 59 (81.5) & 68 (83.8) & $49.0(80.8)$ & 0.73 \\
\hline $\begin{array}{l}\text { Procalcitonin } \mathrm{ng} \cdot \mathrm{mL}^{-1} \text { median (interquartile } \\
\text { range) }\end{array}$ & $0.1(0.25)$ & $0.12(0.21)$ & $0.1(0.37)$ & 0.004 \\
\hline Positive blood culture & $12(6.4)$ & $1(1.2)$ & $11(11.0)$ & 0.204 \\
\hline Positive influenza A/B & 0 & 0 & 0 & 0.593 \\
\hline \multicolumn{5}{|l|}{ Observations } \\
\hline Modified early warning score (MEWS) & $2.6 \pm 1.8$ & $2.9 \pm 1.6$ & $2.3 \pm 1.8$ & 0.605 \\
\hline Temperature ${ }^{\circ} \mathrm{C}$ & $37.5 \pm 1.2$ & $37.8 \pm 1.1$ & $37.2 \pm 1.3$ & 0.174 \\
\hline Respiratory rate $\min ^{-1}$ & $21.2 \pm 6.9$ & $21.9 \pm 7.2$ & $20.7 \pm 6.7$ & 0.888 \\
\hline Oxygen saturation $\%$ & $95.8 \pm 3.2$ & 94.913 .5 & $96.4 \pm 2.8$ & 0.165 \\
\hline Oxygen therapy & $58(0.31)$ & 34 (39.5) & $24(24.0)$ & 0.004 \\
\hline Intubation & $9(4.8)$ & 8 (9.3) & $1(1.0)$ & $<0.001$ \\
\hline \multicolumn{5}{|c|}{$\begin{array}{l}\text { Data are presented as mean } \pm \text { SD or } n(\%) \text {, unless otherwise stated. Statistically significant differences } \\
\text { between the PCR positive and PCR negative groups are emphasised in bold text. ED: emergency } \\
\text { department; IC: intensive care; GOLD: Global Initiative for Chronic Obstructive Lung Disease; CRP: C- } \\
\text { reactive protein. \# : One patient had an indeterminate PCR and no repeat PCR, therefore the total amount } \\
\text { of patients (187) differs from the sum of the PCR positives (86) and PCR negatives (100). }\end{array}$} \\
\hline
\end{tabular}

\section{Discussion}

To our knowledge this is the first study prospectively analysing and comparing the diagnostic accuracy of LUS and chest CT in COVID-19 pneumonia. We found that LUS and CT have similarly good diagnostic accuracy for COVID-19 pneumonia, with AUROC between 0.8 and 0.9. In addition, we found substantial agreement between imaging modalities and good agreement between scanning physicians.

\section{Screening test}

The sensitivity, negative predictive value and negative likelihood ratio found for LUS are promising (table 3). Only seven patients in our cohort had a false negative LUS compared to PCR (supplementary table S4). Three of those patients also did not have a positive CT, indicating they did have COVID-19, but without pulmonary involvement. The reason for the lack of abnormalities could have been the short

\section{TABLE 2 Diagnostic results}

PCR positive

Lung ultrasound: positive
Lung ultrasound: negative
CT: positive
CT: negative

Data are presented as n. CT: computed tomography. 


\section{TABLE 3 Diagnostic accuracy for COVID-19}

\section{Lung ultrasound versus PCR}

Sensitivity \% $(95 \% \mathrm{CI})$

Specificity \% (95\% CI)

Positive likelihood ratio

Negative likelihood ratio

PPV \% $(95 \% \mathrm{CI})$

NPV $\%(95 \% \mathrm{CI})$

Accuracy $\%(95 \% \mathrm{CI})$
$91.9(84.0-96.7)$

$3.2(2.3-4.3)$

$0.1(0.06-0.24)$

$73.2(66.6-78.8)$

$91.0(83.1-95.4)$

$80.7(74.2-86.1)$
$71.0(61.1-79.6)$
CT versus PCR

$88.4(79.7-94.3)$

$82.0(73.1-89.0)$

$4.9(3.2-7.5)$

$0.14(0.08-0.26)$

$80.9(73.4-86.6)$

$89.1(82.0-93.7)$

$85.0(79.0-89.8)$
Lung ultrasound versus COVID-19 diagnosis ${ }^{\#}$

$90.1(82.5-95.2)$

$80.0(69.9-87.9)$

$4.5(2.9-6.9)$

$0.12(0.07-0.2)$

$84.2(77.7-89.2)$

$90.8(81.7-95.6)$

$82.8(76.6-87.9)$

CT: computed tomography; COVID-19: coronavirus disease 2019; PPV: positive predictive value; NPV: negative predictive value; CI: confidence interval. ": According to multidisciplinary team, COVID-19 is highly likely.

duration of symptoms ( 2 days in these patients). Indeed, imaging studies have been shown to be negative in PCR positive patients in the first days of the disease [32, 33]. The four other patients had very mild abnormalities on CT, predominantly in the posterior and inferior zones, that hardly reached the pleura and therefore were (almost) undetectable via LUS. In keeping with what was observed in previous studies, it seems important that the inferior and posterior regions are examined. This can be challenging in obese or immobile patients as illustrated by the fact that one of the false negative ultrasounds occurred in a patient in which the posterior fields could not be examined.

Interestingly, five of the seven "false negative" patients actually did have sonographic COVID-19 features, but only in one unilateral zone, so we regarded those as negative. This might have been a sign of mild or early stage COVID-19 pneumonia. If we had regarded these patients as positive, the AUROC would have increased to 0.87 (95\% CI 0.82-0.93) (supplementary figure S6).

Importantly, none of the patients that were missed on LUS required supplemental oxygen at any stage, nor did they need admission due to COVID-19-related symptoms. Our results demonstrate that LUS can be a safe screening tool for clinically relevant pulmonary involvement in COVID-19 patients who present at the ED.

However, when an alternative diagnosis is lacking, a high post-test probability of COVID-19 without pulmonary involvement may remain, especially when patients present early in the disease course. In those cases, patients should be instructed to home-quarantine and to seek medical help when symptoms worsen. With LUS as an extension of the physical examination, these decisions can be made within minutes of the patient presenting to the ED without any further imaging.

\section{Diagnostic test}

Specificity for LUS was lower than CT: 71.0\% (95\% CI 61.1-79.6) and 82.0\% (95\% CI 73.1-89.0), respectively. The difference in specificity between CT and LUS of $\sim 10 \%$ is consistent with what has previously been reported in the literature $[24,26,27]$.

One explanation for the low specificity of both modalities could be the lack of sensitivity of the PCR. This automatically generates more "false positives" $[32,33]$. We therefore also compared LUS with the MDT diagnosis. In our cohort 29 patients had a positive LUS and negative PCR. Twelve of them were eventually diagnosed with COVID-19 by the MDT. This led to a marked increase in specificity and PLR. Due to incorporation bias (incorporation of the CT results in the MDT diagnosis), CT should not be compared to the MDT diagnosis as this would overestimate accuracy.

Another reason for the lower specificity is that the sonographic features of COVID-19 are not exclusive to COVID-19. They are also observed in other aetiologies like (viral or atypical) pneumonia, interstitial lung disease (ILD), acute respiratory distress syndrome (ARDS), heart failure and atelectasis. However, this is also the case for CT, albeit to a lesser extent $[9,12,32,33]$. The most common diagnoses in patients with a false positive LUS were: pneumonia of another source (6), atelectasis in bedridden patients (5) and drug-induced pneumonitis (3). There were no patients with underlying ILD in our cohort. When the prevalence of COVID-19 decreases and/or the prevalence of diseases which produce similar findings (e.g. ILD or influenza) increases, the positive predictive value of LUS will probably decrease concomitantly. More research has to be done to assess whether LUS can distinguish COVID-19 from similar disease processes.

\section{Strengths}

The results of this study are in line with the literature on diagnostic accuracy of LUS in acute respiratory failure caused by different aetiologies ranging from pneumonia to ARDS [24, 26, 27, 29, 30, 34, 35] 
Almost all LUS were performed by acute internal medicine registrars with modest LUS experience, which underscores the applicability of LUS in a real-life setting.

Advantages of LUS over CT include: ease of use, affordability, repeatability and avoidance of radiation $[13,14,21-23]$. LUS can be used in (unstable) patients without the need to transport them. This reduces exposure in healthcare personnel and equipment, which minimises wasting of scarce resources and potentially prevents nosocomial spread of infection $[9,12,18,23]$. In addition, by integrating imaging into clinical decision-making at the bedside, LUS can reduce time to diagnosis and treatment at the ED [28, 30]. One can also reduce diagnostic uncertainty by scanning other structures, such as the inferior vena cava, heart and deep venous system of the lower extremities [24, 28-30]. If uncertainty still remains about the cause of respiratory symptoms or hypoxia, the treating physician can always employ additional conventional tests like CT.

COVID-19 has laid bare health disparities along socioeconomic, racial, cultural and ethnic lines across and within nations $[2,3]$. CT is costly and might not be readily available, even in high-income countries $[8,20]$. LUS is an affordable alternative, especially when handheld devices are used. It may be used in any care setting, further reducing barriers to adequate care $[17,19]$. It has even been shown that it can be accurately performed by non-physicians who are guided remotely, so patients could be screened extramurally [36]. LUS has a steep learning curve, for physicians and other (para)medical personnel, and the basics can be learned in under $2 \mathrm{~h}$ [37-39].

\section{Limitations/bias}

Our study has some important limitations. First, this was an observational study; no blinding, randomisation or power calculation was performed. Second, our cohort was a convenience sample. We have tried to enrol every adult patient with suspected COVID-19 referred to internal medicine when a certified sonographer was present. We therefore feel that our cohort is random and representative, and the chance of selection bias is minimal. Our cohort seems consistent with what is found in the literature in terms of mean duration of symptoms, comorbidity and mortality. We had a high number of patients with malignancies because all three hospitals are tertiary oncology centres. Third, the radiologists and scanning physicians were aware of the patients' history and clinical pictures. While this may have led to some bias, clinical tests should preferably be guided by thorough clinical assessment. The integration of imaging with clinical assessment in real-time is one of the main advantages of bedside ultrasound. Blinding sonographers for research purposes is therefore not desirable as the results would not be generalisable to daily practice.

\section{Conclusion}

Our study shows that the diagnostic accuracy of LUS is comparable to CT. We demonstrated that LUS can help in triage by excluding clinically relevant COVID-19 pneumonia at the ED and may aid in diagnosis of COVID-19 in a high-prevalence setting. It may prove especially useful in situations where CT or PCR results are not readily available. We advocate the use of LUS as an extension of the physical examination and encourage setting up training programmes worldwide so this tool can be used during and subsequent to the pandemic. We also suggest that further studies be conducted in different settings to validate our findings.

Conflict of interest: None declared.

\section{References}

1 Johns Hopkins Coronavirus Resource Center. New Cases of COVID-19 in World Countries. Date last accessed: June 9, 2020. Date last updated June 9, 2020. https://coronavirus.jhu.edu/data/new-cases

2 Centers for Disease Control and Prevention. Coronavirus Disease 2019 (COVID-19). Date last accessed: May 21, 2020. Date last updated: May 21, 2020. www.cdc.gov/coronavirus/2019-ncov/php/public-health-recommendations. html

3 Kavanagh MM, Erondu NA, Tomori O, et al. Access to lifesaving medical resources for African countries: COVID-19 testing and response, ethics, and politics. Lancet 2020; 395: 1735-1738.

4 Wang W, Xu Y, Gao R, et al. Detection of SARS-CoV-2 in different types of clinical specimens. JAMA 2020; 323: $1843-1844$.

5 Abbasi J. The promise and peril of antibody testing for COVID-19. JAMA 2020; 323: 1881-1883.

6 Guan W, Ni Z, Hu Y, et al. Clinical characteristics of coronavirus disease 2019 in China. N Engl J Med 2020; 382: $1708-1720$.

7 Zhou F, Yu T, Du R, et al. Clinical course and risk factors for mortality of adult inpatients with COVID-19 in Wuhan, China: a retrospective cohort study. Lancet 2020; 395: 1054-1062.

8 Use of chest imaging in COVID-19 Date last accessed: June 13, 2020. Date last updated June 11, 2020. www.who. int/publications-detail-redirect/use-of-chest-imaging-in-covid-19

9 Rubin GD, Haramati LB, Kanne JP, et al. The role of chest imaging in patient management during the COVID-19 pandemic: a multinational consensus statement from the Fleischner Society. Radiology 2020; 296: 172-180. 
10 Simpson S, Kay FU, Abbara S, et al. Radiological Society of North America Expert Consensus Statement on Reporting Chest CT Findings Related to COVID-19. Endorsed by the Society of Thoracic Radiology, the American College of Radiology, and RSNA. Radiol Cardiothorac Imaging 2020; in press [https://doi.org/10.1148/ryct. 2020200152].

11 The Royal College of Radiologists. The role of CT in patients suspected with COVID-19 infection. Date last accessed: July 8, 2020. Date last updated 12 March, 2020. www.rcr.ac.uk/college/coronavirus-covid-19-what-rcrdoing/clinical-information/role-ct-chest/role-ct-patients

12 Hope MD, Raptis CA, Shah A, et al. A role for CT in COVID-19? What data really tell us so far. Lancet 2020 395: 1189-1190.

13 Buonsenso D, Pata D, Chiaretti A. COVID-19 outbreak: less stethoscope, more ultrasound. Lancet Respir Med 2020; 8: e27.

14 Cheung JC-H, Lam KN. POCUS in COVID-19: pearls and pitfalls. Lancet Respir Med 2020; 8: e34.

15 https://bestpractice.bmj.com/topics/en-us/3000168/investigations. Date last accessed: June 12, 2020; Date last updated: June 12, 2020.

16 https://www.bmus.org/static/uploads/resources/COVID19_Lung_Ultrasound_BMUS.pdf. Date last accessed: 4 April 2020. Date last updated: March, 2020.

17 Sofia S, Boccatonda A, Montanari M, et al. Thoracic ultrasound and SARS-COVID-19: a pictorial essay. J Ultrasound 2020; 23: 217-221.

18 Volpicelli G, Lamorte A, Villén T. What's new in lung ultrasound during the COVID-19 pandemic. Intensive Care Med 2020; 46: 1445-1448.

19 Soldati G, Smargiassi A, Inchingolo R, et al. Is there a role for lung ultrasound during the COVID-19 pandemic? Clinical Letters. J Ultrasound Med 2020; 39: 1459-1462.

20 Poggiali E, Dacrema A, Bastoni D, et al. Can lung US help critical care clinicians in the early diagnosis of novel coronavirus (COVID-19) pneumonia? Radiology 2020; 295: E6.

21 Huang Y, Wang S, Liu Y, et al. A preliminary study on the ultrasonic manifestations of peripulmonary lesions of non-critical novel coronavirus pneumonia (COVID-19). SSRN 2020; preprint [https://dx.doi.org/10.2139/ssrn. 3544750].

22 Chinese Critical Care Ultrasound Study Group (CCUSG), Peng Q-Y, Wang X-T, et al. Findings of lung ultrasonography of novel corona virus pneumonia during the 2019-2020 epidemic. Intensive Care Med 2020; 46 : 849-850.

23 Smith MJ, Hayward SA, Innes SM, et al. Point-of-care lung ultrasound in patients with COVID -19 - a narrative review. Anaesthesia 2020; 75: 1096-1104.

24 Lichtenstein DA. BLUE-Protocol and FALLS-Protocol. Chest 2015; 147: 1659-1670.

25 Ye X, Xiao H, Chen B, et al. Accuracy of lung ultrasonography versus chest radiography for the diagnosis of adult community-acquired pneumonia: review of the literature and meta-analysis. PLoS One 2015; 10: e0130066.

26 Orso D, Guglielmo N, Copetti R. Lung ultrasound in diagnosing pneumonia in the emergency department: a systematic review and meta-analysis. Eur J Emerg Med 2018; 25: 312-321.

27 Staub LJ, Mazzali Biscaro RR, Kaszubowski E, et al. Lung ultrasound for the emergency diagnosis of pneumonia, acute heart failure, and exacerbations of chronic obstructive pulmonary disease/asthma in adults: a systematic review and meta-analysis. J Emerg Med 2019; 56: 53-69.

28 Laursen CB, Sloth E, Lassen AT, et al. Point-of-care ultrasonography in patients admitted with respiratory symptoms: a single-blind, randomised controlled trial. Lancet Respir Med 2014; 2: 638-646.

29 Pivetta E, Goffi A, Nazerian P, et al. Lung ultrasound integrated with clinical assessment for the diagnosis of acute decompensated heart failure in the emergency department: a randomized controlled trial. Eur J Heart Fail 2019; 21: 754-766.

30 Smallwood N, Dachsel M. Point-of-care ultrasound (POCUS): unnecessary gadgetry or evidence-based medicine? Clin Med 2018; 18: 219-224.

31 RIVM. Epidemiologische situatie COVID-19 in Nederland 13 juni 2020.Date last accessed: June 14, 2020. Date last updated: June 12, 2020. www.rivm.nl/documenten/epidemiologische-situatie-covid-19-in-nederland-12-juni-2020

32 Prokop M, van Everdingen W, van Rees Vellinga T, et al. CO-RADS - A categorical CT assessment scheme for patients with suspected COVID-19: definition and evaluation. Radiology 2020; 296: E97-E104.

33 Ai T, Yang Z, Hou H, et al. Correlation of chest CT and RT-PCR testing in coronavirus disease 2019 (COVID-19) in China: a report of 1014 Cases. Radiology 2020; 296: E32-E40.

34 Lee FCY. Lung ultrasound - a primary survey of the acutely dyspneic patient. J Intensive Care 2016; 4: 57.

35 Mojoli F, Bouhemad B, Mongodi S, et al. Lung ultrasound for critically ill patients. Am J Respir Crit Care Med 2019; 199: 701-714.

36 Kirkpatrick AW, McKee JL. Lung ultrasonography in a woman with COVID-19: this examination could be remote. CMAJ 2020; 192: E435-E435.

37 Arbelot C, Dexheimer Neto FL, Gao Y, et al. Lung ultrasound in emergency and critically ill patients: number of supervised exams to reach basic competence. Anesthesiology 2020; 132: 899-907.

38 See KC, Ong V, Wong SH, et al. Lung ultrasound training: curriculum implementation and learning trajectory among respiratory therapists. Intensive Care Med 2016; 42: 63-71.

39 Tulleken AM, Gelissen H, Lust E, et al. UltraNurse: teaching point-of-care ultrasound to intensive care nurses. Intensive Care Med 2019; 45: 727-729. 